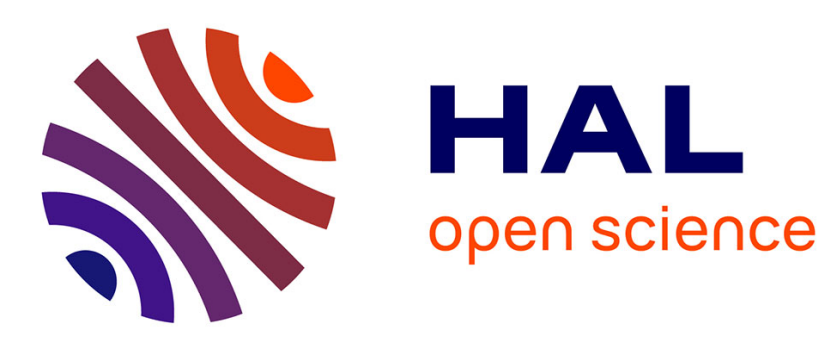

\title{
Interaction Energy of a Charged Medium and its EM Field in a Curved Spacetime
}

\author{
Mayeul Arminjon
}

\section{To cite this version:}

Mayeul Arminjon. Interaction Energy of a Charged Medium and its EM Field in a Curved Spacetime. Proceedings of the Twentieth International Conference on Geometry, Integrability and Quantization, Ivaïlo M. Mladenov, Jun 2018, Varna, Bulgaria. pp.88-98. hal-01881100

\section{HAL Id: hal-01881100 https://hal.science/hal-01881100}

Submitted on 25 Sep 2018

HAL is a multi-disciplinary open access archive for the deposit and dissemination of scientific research documents, whether they are published or not. The documents may come from teaching and research institutions in France or abroad, or from public or private research centers.
L'archive ouverte pluridisciplinaire HAL, est destinée au dépôt et à la diffusion de documents scientifiques de niveau recherche, publiés ou non, émanant des établissements d'enseignement et de recherche français ou étrangers, des laboratoires publics ou privés. 


\title{
Interaction Energy of a Charged Medium and its EM Field in a Curved Spacetime
}

\author{
Mayeul Arminjon \\ Univ. Grenoble Alpes, CNRS, Grenoble INP, 3SR lab., F-38000 Grenoble, France
}

\begin{abstract}
In the electrodynamics of special relativity (SR) or general relativity (GR), the energy tensors of the charged medium and its EM field add to give the total energy tensor that obeys the dynamical equation without external force. In the investigated scalar theory of gravitation ("SET"), this assumption leads to charge non-conservation, hence an additional, "interaction" energy tensor $\boldsymbol{T}_{\text {inter }}$ has to be postulated. The present work aims at constraining this tensor. First we study the independent equations of electrodynamics and their number, beginning with SR and GR. As in SR and GR, the system of electrodynamics of SET is closed in the absence of $\boldsymbol{T}_{\text {inter }}$. Hence, with $\boldsymbol{T}_{\text {inter }}$, at least one additional equation must be provided. This is done by assuming that $\boldsymbol{T}_{\text {inter }}$ is Lorentz-invariant in the situation of SR. We derive equations allowing one in principle to compute $\boldsymbol{T}_{\text {inter }}$ in a given gravitational plus EM field. $\boldsymbol{T}_{\text {inter }}$ may contribute to the dark matter.
\end{abstract}

MSC: 78A25, 83A05, 83C50, 83D05

Keywords: Maxwell equations, special relativity, general relativity, alternative theory of gravitation, preferred reference frame.

\section{Introduction}

The main motivation for the work summarized in this paper is to develop a consistent electrodynamics in an alternative theory of gravity with a preferred reference frame: "the scalar ether theory", or in short SET [4, 5]. In turn the motivations for SET, which have been exposed in detail elsewhere 
[7, are essentially as follows. (i) Special relativity (SR) can be interpreted within classical concepts of space and time, thus keeping a "preferred" simultaneity. This is the Lorentz-Poincaré version of SR, otherwise named "the Lorentz ether theory". SET, which is a relativistic theory of gravitation with a curved Lorentzian metric, extends that alternative version or interpretation of SR to the general situation with gravitation. (ii) SET has a physical interpretation for gravity: a pressure force (Archimedes' thrust in a fluid "ether") [4. (iii) Some problems of general relativity (GR) are avoided in SET: the singularities (in the gravitational collapse [1] and in cosmology as well [3]); the dark energy problem (at least in the sense that SET necessarily predicts an acceleration of the cosmic expansion [3]); the interpretation of the gauge condition (because there is no gauge condition in SET); the non-uniqueness problem of the covariant Dirac theory (because in SET's preferred reference frame, the metric does have the diagonal spatially-isotropic form necessary to implement the "radical" and simple solution proposed in Ref. [6]).

In GR, the equations of electrodynamics rewrite those of SR by using the "comma goes to semicolon" rule: the partial derivatives are replaced by covariant derivatives [16]. To use this rule is not possible in SET, essentially because its dynamical equation does not generally coincide with that of GR and other metric theories of gravity, namely $T_{; \nu}^{\lambda \nu}=0$ - whereas, precisely, that equation rewrites the equation $T_{, \nu}^{\lambda \nu}=0$, that is valid in SR, by using the rule just mentioned. In SET, the first Maxwell group (of equations) stays unchanged. As to the second group, it was got by applying the dynamical equation of SET to a charged medium in the presence of the Lorentz force, assuming that - as is the case in GR:

(i) The total energy(-momentum-stress) tensor $\boldsymbol{T}$ is the sum of the energy tensors of the charged medium and the electromagnetic (EM) field:

$$
\boldsymbol{T}=\boldsymbol{T}_{\text {charged medium }}+\boldsymbol{T}_{\text {field }} .
$$

(ii) The total energy tensor $\boldsymbol{T}$ obeys the dynamical equation of the theory, without any non-gravitational force.

Assumptions (i) and (ii) lead uniquely to a form of Maxwell's second group in SET, and this form turns out to predict production/destruction of electric charge [7]. Moreover, it also turns out that this is at unrealistic 
rates [8]. For this reason, and also for more theoretical reasons, that form of Maxwell's second group in SET has to be discarded [8]. Whereas Assumption (ii) is quite essential to the theory [5], Assumption (i) is not necessary and may be abandoned. This amounts to introducing an "interaction" energy tensor $\boldsymbol{T}_{\text {inter }}$ such that

$$
\boldsymbol{T}=\boldsymbol{T}_{\text {charged medium }}+\boldsymbol{T}_{\text {field }}+\underline{\boldsymbol{T}_{\text {inter }}} .
$$

The aim of this work, therefore, was to constrain the form of $\boldsymbol{T}_{\text {inter }}$ and to derive equations that should enable one later to calculate it in a realistic gravitational plus EM field. (A more detailed account of this same work has been given in Ref. [9]. So here many derivations shall be skipped but, hopefully, clear explanations will be provided for the basic facts.) This needs paying due attention to the independent equations in electrodynamics and to their number. Let us begin with standard theory: SR and GR.

\section{Maxwell Equations in Standard Theory}

Maxwell's first group of equations for the EM antisymmetric field tensor $F_{\lambda \nu}$ is:

$$
F_{\lambda \nu, \rho}+F_{\nu \rho, \lambda}+F_{\rho \lambda, \nu} \equiv F_{\lambda \nu ; \rho}+F_{\nu \rho ; \lambda}+F_{\rho \lambda ; \nu}=0 .
$$

(The first equality is indeed an identity.) It can be rewritten as

$$
M_{\lambda \nu \rho}:=F_{\lambda \nu, \rho}+F_{\nu \rho, \lambda}+F_{\rho \lambda, \nu}=0 .
$$

This is fully antisymmetric, hence four equations are linearly independent, e.g.

$$
M_{012}=0, \quad M_{013}=0, \quad M_{023}=0, \quad M_{123}=0 .
$$

Maxwell's second group in SR and in GR has also four equations:

$$
F_{; \nu}^{\lambda \nu}=-\mu_{0} J^{\lambda} \quad(\lambda=0, \ldots, 3) .
$$

(Here, $J^{\lambda}$ is the 4-current. We are using the SI units, as opposed to Gauss units.) 


\subsection{Independent Equations in Standard Theory (Given Source)}

Assuming that the source $J^{\lambda}$ is given, we thus have $4+4=\mathbf{8}$ equations for $\mathbf{6}$ unknowns $F_{\lambda \nu}(0 \leq \lambda<\nu \leq 3)$ (or equivalently for the six components of the electric and magnetic spatial vector fields $\mathbf{E}$ and $\mathbf{B})$. As is well known, those eight equations are nevertheless needed, e.g. one cannot remove the Gauss equation for magnetism, $\operatorname{div} \mathbf{B}=0$ [13, 15, 18]. This can be explained simply [9] by noting two differential identities of Maxwell's system of equations. The first one is [12, 9]:

$$
e^{\lambda \nu \rho \sigma} M_{\lambda \nu \rho ; \sigma} \equiv 0
$$

and is a differential identity of the first Maxwell group (4). (Here $e^{\lambda \nu \rho \sigma}$ is the standard fully antisymmetric tensor.) Using the total antisymmetry of the $M_{\lambda \nu \rho}$ tensor, it can be rewritten in terms of $M_{012}, M_{013}, M_{023}, M_{123}$ only. It is hence a differential identity of (5) as well. For the second group (6), using the identity $F_{; \nu ; \lambda}^{\lambda \nu} \equiv 0$, we obtain first the charge conservation as a compatibility condition of Eq. (6):

$$
J_{; \lambda}^{\lambda}=0 .
$$

If the condition (8) is satisfied, then, using again the identity $F_{; \nu ; \lambda}^{\lambda \nu} \equiv 0$, we get a differential identity for the second group (6):

$$
\left(F_{; \nu}^{\lambda \nu}+\mu_{0} J^{\lambda}\right)_{; \lambda} \equiv 0
$$

Note that, by definition of a differential identity, Eqs. (7) and (9) are valid whether or not any of the two groups of equations (5) and (6) is satisfied. So the differential identity (7) reduces the number of independent equations in Eq. (5) from 4 to 3, and (9) does the same for (6) — thus reducing the number of independent equations in the Maxwell system, Eqs. (5) and (6), from 8 to 6 - that is the number of the unknowns. But on the other hand, because (7) and (9) are differential identities and not linear dependence relations between the equations themselves of the systems (5) and (6), they do not allow one to remove any among these equations. Thus (7) does not

allow one to remove any among the four equations in the system (5), and (9) does not allow one to remove any among the four equations in the system (6). 


\subsection{Independent Equations in Standard Theory (Gen- eral Case)}

If the 4-current $\mathbf{J}$ is not given, we have at least $\mathbf{5}$ unknowns more: the charge density of the charged continuum $\rho_{\mathrm{el}}$, its 3 -velocity field $\mathbf{v}$, plus the other state parameters of the continuum, say only its proper rest-mass density $\rho^{*}$. (The data of $\mathbf{J}$ is equivalent to that of $\rho_{\mathrm{el}}$ and $\mathbf{v}$, provided one sets the assumption that the charges are bound to the charged continuum.) As additional equation with respect to the Maxwell equations (5) and (6), we have only the dynamical equation for the charged continuum:

$$
T_{\text {chg } ; \nu}^{\mu \nu}=F_{\lambda}^{\mu} J^{\lambda} .
$$

(This implies the mass conservation at least for an isentropic fluid.) Thus we have only 4 scalar equations more. However, now Eq. (9) applies provided that Maxwell's second group (6) is satisfied by the two unknown fields $\boldsymbol{F}$ and J. Therefore, Eq. (9) is not a differential identity of the new system [(5), (6), and (10)]: it applies only on its solution space. Thus only the differential identity (7) counts as a dependence relation, so we have $4+4+4-1=11$ independent equations for $6+5=11$ unknowns.

\section{Interaction Energy Tensor in SET}

As was recalled in the Introduction, previous work [8] showed that in SET we must consider

$$
\boldsymbol{T}=\boldsymbol{T}_{\text {chg }}+\boldsymbol{T}_{\text {field }}+\boldsymbol{T}_{\text {inter }} \neq \boldsymbol{T}_{\text {chg }}+\boldsymbol{T}_{\text {field }}
$$

in the dynamical equation. (Here chg $=$ charged medium.)

\subsection{Dynamical Equations in SET}

The dynamical equation for the total energy tensor in SET is [2]:

$$
T_{; \nu}^{\mu \nu}=b^{\mu}(\boldsymbol{T}),
$$

where

$$
b^{0}(\boldsymbol{T}):=\frac{\gamma^{00}}{2} g_{i j, 0} T^{i j}, \quad b^{i}(\boldsymbol{T}):=\frac{1}{2} g^{i j} g_{j k, 0} T^{0 k},
$$


with $\gamma$ the spacetime metric, and where $\boldsymbol{g}$ is the spatial metric [11, 14, 17] in the preferred reference frame $\mathcal{E}$ assumed by SET. Note: Consistently with the preferred-frame character of the theory, the equations of SET usually are spatially-covariant only, the coordinate time $T:=x^{0} / c$ being thus a preferred time [5]. However, Eq. (12) and the definition (13) are covariant also under changes of the time coordinate having the form $x^{\prime 0}=\phi\left(x^{0}\right)$ [5].

For a continuous medium in the presence of a field of external nongravitational 3 -force $\mathbf{f}=\left(f^{i}\right)(i=1,2,3)$, the dynamical equation is [7]

$$
T_{\text {medium } ; \nu}^{\mu \nu}=b^{\mu}\left(\boldsymbol{T}_{\text {medium }}\right)+f^{\mu}, \quad f^{0}:=\frac{\mathbf{f} \cdot \mathbf{v}}{c \beta},
$$

where $\beta:=\sqrt{\gamma_{00}}$ and $\mathbf{v}$ is the 3 -velocity field defined with the local time. For a charged medium $\left(\boldsymbol{T}_{\text {medium }}=\boldsymbol{T}_{\text {chg }}\right)$ subjected to an EM field, we get $f^{\mu}=F_{\nu}^{\mu} J^{\nu}$ [7], so (14) is

$$
T_{\text {chg } ; \nu}^{\mu \nu}=b^{\mu}\left(\boldsymbol{T}_{\text {chg }}\right)+F_{\nu}^{\mu} J^{\nu} .
$$

\subsection{Independent Equations and Unknowns for SET}

The independent equations have the same structure as in GR: $2^{2}$

- Maxwell's first group (5):

4 equations

- Dynamical equation for the total energy tensor (12): 4 equations

- Dynamical equation for the charged medium (15): 4 equations

- Minus one differential identity $e^{\lambda \nu \rho \sigma} M_{\lambda \nu \rho ; \sigma} \equiv 0$ : $\quad-1$ equation,

thus 11 independent equations. (For GR, Eqs. (12) and (15) are replaced by equations in which the $b^{\mu}$ term is replaced by zero.)

The list of the independent unknowns is also close to that in GR:

- EM field $F_{\mu \nu}(0 \leq \mu<\nu \leq 3)$ :

6 unknowns

\footnotetext{
${ }^{2}$ Indeed, in GR, Maxwell's second group (6) can be exchanged with the dynamical equation for the charged medium in the list below, by using the additivity (1). See Ref. [9].
} 
- 4-current J:

- proper rest-mass density $\rho^{*}$ :

- $p l u s$ at least one new field to define $\boldsymbol{T}_{\text {inter }}$
4 unknowns

1 unknown

$\geq 1$ unknown

Hence we have at least 12 independent unknowns, so that at least one equation more is needed.

\subsection{Dynamical Equation with Energy Interaction Ten- sor}

Using the general decomposition of the total energy tensor $\boldsymbol{T}(11)$ and the linearity of $b^{\mu}$ (Eq. (13)) with respect to the energy tensor in the dynamical equation (12) for $\boldsymbol{T}$ in SET, one sees that this latter equation is equivalent to:

$$
T_{\text {field } ; \nu}^{\mu \nu}=b^{\mu}\left(\boldsymbol{T}_{\text {field }}\right)+b^{\mu}\left(\boldsymbol{T}_{\text {chg }}\right)-T_{\text {chg } ; \nu}^{\mu \nu}+b^{\mu}\left(\boldsymbol{T}_{\text {inter }}\right)-T_{\text {inter } ; \nu}^{\mu \nu} .
$$

Maxwell's first group implies an identity for the energy tensor of the EM field [7]:

$$
\mu_{0} T_{\text {field } ; \nu}^{\mu \nu} \equiv F_{\lambda}^{\mu} F_{; \nu}^{\lambda \nu} .
$$

By using this and the dynamical equation (15) for the charged medium, (16) rewrites as

$$
F_{\lambda}^{\mu} F_{; \nu}^{\lambda \nu}=\mu_{0}\left[b^{\mu}\left(\boldsymbol{T}_{\text {field }}\right)-F_{\nu}^{\mu} J^{\nu}-\delta^{\mu}\right],
$$

where

$$
\delta_{\mu}:=T_{\text {inter } \mu ; \nu}^{\nu}-b_{\mu}\left(\boldsymbol{T}_{\text {inter }}\right) .
$$

If the matrix $\left(F_{\lambda}^{\mu}\right)$ is invertible, ${ }^{3}$ Eq. (18) becomes

$$
F_{; \nu}^{\mu \nu}=\mu_{0}\left[G_{\nu}^{\mu}\left(b^{\nu}\left(\boldsymbol{T}_{\text {field }}\right)-\delta^{\nu}\right)-J^{\mu}\right],
$$

3 This is equivalent to $\mathbf{E . B} \neq 0$ [7]. It is not valid for the simple and most known solutions of the Maxwell equations: purely electric or purely magnetic fields, usual plane waves, dipole field [8]. However, it is generally true, because a real EM field is a combination of simple solutions and, if one adds two solutions $\left(\mathbf{E}_{1}, \mathbf{B}_{1}\right),\left(\mathbf{E}_{2}, \mathbf{B}_{2}\right)$ of the standard Maxwell equations such that $\mathbf{E}_{1} \cdot \mathbf{B}_{1}=0$ and $\mathbf{E}_{2} \cdot \mathbf{B}_{2}=0$, then $\left(\mathbf{E}_{1}+\mathbf{E}_{2}, \mathbf{B}_{1}+\mathbf{B}_{2}\right)$ is also a solution, but then

$$
\left(\mathbf{E}_{1}+\mathbf{E}_{2}\right) \cdot\left(\mathbf{B}_{1}+\mathbf{B}_{2}\right)=\mathbf{E}_{1} \cdot \mathbf{B}_{2}+\mathbf{E}_{2} \cdot \mathbf{B}_{1},
$$

which generally is not zero. In short, E.B depends non-linearly on the field $(\mathbf{E}, \mathbf{B})$. 
with $\left(G^{\mu}{ }_{\nu}\right):=\left(F^{\mu}{ }_{\nu}\right)^{-1}$. Using once again the identity $F_{; \nu ; \lambda}^{\lambda \nu} \equiv 0$, we deduce from (21) that

$$
J_{; \mu}^{\mu}=\left[G_{\nu}^{\mu}\left(b^{\nu}\left(\boldsymbol{T}_{\text {field }}\right)-\delta^{\nu}\right)\right]_{; \mu} .
$$

\subsection{Form of the Interaction Energy Tensor}

In SR, the interaction energy tensor $\boldsymbol{T}_{\text {inter }}$ is zero. Since SET should coincide with SR in the situation without gravitational field, one wishes that in this situation one should have $\boldsymbol{T}_{\text {inter }}=\mathbf{0}$ in SET. Of course we cannot directly impose that $\boldsymbol{T}_{\text {inter }}=\mathbf{0}$ in the system of equations, because this would lead us again to the first version [7] of the electrodynamics of SET. In particular, it would lead us to Eq. (22) with $\delta^{\nu} \equiv 0$, which is the equation for charge production/destruction in that former version, that has been discarded [8]. However, we may impose that $\boldsymbol{T}_{\text {inter }}$ should be Lorentz-invariant in a Minkowski spacetime. This is true if and only if [10] we have when the metric $\gamma$ is Minkowski's $\left(\gamma_{\mu \nu}=\eta_{\mu \nu}\right.$ in Cartesian coordinates):

$$
T_{\text {inter } \mu \nu}=p \eta_{\mu \nu} \quad \text { (situation of SR), }
$$

with some scalar field $p$. This is equivalent to:

$$
T_{\text {inter } \quad \nu}^{\mu}:=\eta^{\mu \rho} p \eta_{\rho \nu}=p \delta_{\nu}^{\mu} \quad \text { (situation of SR). }
$$

The definition

$$
T_{\text {inter } \nu}^{\mu}:=p \delta_{\nu}^{\mu}, \quad \text { or } \quad\left(T_{\text {inter }}\right)_{\mu \nu}:=p \gamma_{\mu \nu},
$$

thus got in Cartesian coordinates in a Minkowski spacetime, is actually generally-covariant. Therefore, we adopt (25) for the general case.

\subsection{Closing the Electrodynamics of SET with Charge Conservation}

With the "scalar" interaction energy tensor (25), we have just one unknown more: the field $p$. Thus we may add the charge conservation as a unique new scalar equation to close the system of equations of electrodynamics of SET, Eqs. (5), (12), 15) (see the first list in Subsect. 3.2). In view of Eq. (22), this consists in adding the scalar equation

$$
\left[G^{\mu \nu}\left(b_{\nu}\left(\boldsymbol{T}_{\text {field }}\right)-\delta_{\nu}\right)\right]_{; \mu}=0 .
$$


In contrast, when in the previous work [7] we assumed the additivity of energy tensors (as in GR), i.e., $\boldsymbol{T}_{\text {inter }}=\mathbf{0}$, then the system [(5), (12), (15)] alone was closed. Thus in the latter case, charge conservation could not be imposed. In fact a significant charge production/destruction was then predicted by Eq. (22) with $\delta^{\nu} \equiv 0$ [8], which is not observed.

The dynamical equations of SET: Eq. 12 for the total energy tensor and Eq. (15) for the energy tensor of the charged medium, coincide for a constant gravitational field with the corresponding equations of GR. Moreover, the first Maxwell group (5) is the same in SET and in GR, in the most general situation and hence in particular for a constant gravitational field. Finally, the definition (25) of the interaction energy tensor gives in general [9] for $\delta_{\mu}$ defined in Eq. (19):

$$
\begin{gathered}
\delta_{0}=p_{, 0}-3 p \beta_{, 0} \beta^{-1} \\
\delta_{i}=p_{, i},
\end{gathered}
$$

and this is zero in a constant gravitational field $\left(\beta_{, 0}=0\right)$, provided that $p_{, \mu}=0$ in that case. Thus, in a constant gravitational field, all equations of the system [(5), (12), (15), 26)] are solved by $p \equiv$ Constant and with the set of the other fields (see the second list of Subsect. 3.2) being the solution of the system of electrodynamics of GR for the given boundary conditions. Therefore, assuming uniqueness of the solution (as is the case for standard electrodynamics), this is the solution. We thus find that the field $p$ is constant in a constant gravitational field, and hence in particular in the situation of SR. Moreover, that constant must be zero in fact, because, at a very large distance from any material body, also the total energy tensor $\boldsymbol{T}$ given by Eq. (11) must be zero, as are $\boldsymbol{T}_{\text {chg }}$ and (asymptotically) $\boldsymbol{T}_{\text {field }}$. It follows that the additivity (1) of the energy tensors is recovered for a constant gravitational field, and hence in particular in the situation of SR.

As shown by Eqs. (27)-(28), $\delta_{\mu}$ depends only on $p$ (and on the gravitational field $\beta$ ). Thus, in general, Eq. (26) should determine the field $p$ in a given gravitational plus EM field. We now show how to do that in a weak gravitational field. 


\section{Determining the Interaction Energy in a Weak Gravitational Field}

\subsection{Weak Gravitational Field}

An asymptotic framework was developed for an EM field in a weak and slowly varying gravitational field [8]. Essentially: we conceptually associate with the given system $\mathrm{S}$ a family $\left(\mathrm{S}_{\lambda}\right)$ of systems, depending on the weakgravitational-field parameter $\lambda \rightarrow 0$, with $\lambda=1 / c^{2}$ in specific $\lambda$-dependent units of mass and time. Of course the EM field is not assumed weak nor slowly varying. Then we write Taylor expansions w.r.t. $\lambda$; e.g., for the EM field tensor:

$$
\boldsymbol{F}=c^{n}\left(\stackrel{0}{\boldsymbol{F}}+c^{-2} \stackrel{1}{\boldsymbol{F}}+O\left(c^{-4}\right)\right),
$$

where $n$ could be any integer. And

$$
p=c^{2 n-5}\left(\stackrel{0}{p}+c^{-2} \stackrel{1}{p}+O\left(c^{-4}\right)\right),
$$

for the "interaction scalar" field $p$ with $\boldsymbol{T}_{\text {inter }}=p \boldsymbol{\gamma}$, Eq. (25). (The order $2 n-5$ follows from the equation for charge conservation, Eq. (26) [9].)

\subsection{Interaction Scalar in a Weak Gravitational Field}

Using such asymptotic expansions in the equation of charge conservation in the form of Eq. (26), we obtained an advection equation with a given source, for the first-approximation field $p_{1}:=c^{2 n-5} \stackrel{0}{p} \quad[9]$ :

$$
\partial_{T} p_{1}+u^{j} \partial_{j} p_{1}=S
$$

where

$$
S:=\frac{c^{-2}\left(e^{i} \partial_{T} U\right)_{, i}}{k^{0}}
$$

and

$$
u^{j}:=\frac{c k^{j}}{k^{0}}
$$

Here $U$ is the Newtonian gravitational potential. On the other hand, $e^{i}$ and $k^{\mu}$ depend only on the first-approximation EM field $(\mathbf{E}, \mathbf{B})$, that obeys the 
standard flat-spacetime Maxwell equations [8, 9]. In particular,

$$
k^{0}=\frac{-c}{(\mathbf{E} . \mathbf{B})^{2}} \text { B. }(\nabla(\mathbf{E} . \mathbf{B})),
$$

which must be well defined and non-zero. (Cf. Footnote 3 .)

We then derive easily the explicit solution of Eq. (31) by integration along characteristics [9]. Given an arbitrary event $\left(T_{0}, \mathbf{x}_{0}\right)$, let $T \mapsto \mathbf{x}(T)=$ $\mathcal{C}_{T_{0} \mathbf{x}_{0}}(T)$ be the (unique) solution of the ODE

$$
\frac{\mathrm{d} \mathbf{x}}{\mathrm{d} T}=\mathbf{u}(T, \mathbf{x}), \quad \mathbf{x}\left(T_{0}\right)=\mathbf{x}_{0}
$$

Thus $\mathcal{C}_{T_{0} \mathbf{x}_{0}}$ is the characteristic curve passing at $\left(T_{0}, \mathbf{x}_{0}\right)$. The solution $p_{1}$ of (31) on that curve is given uniquely by:

$$
p_{1}\left(T, \mathcal{C}_{T_{0} \mathbf{x}_{0}}(T)\right)-p_{1}\left(T_{0}, \mathbf{x}_{0}\right)=\int_{T_{0}}^{T} S\left(t, \mathcal{C}_{T_{0} \mathbf{x}_{0}}(t)\right) \mathrm{d} t .
$$

If at time $T_{0}$ the position $\mathbf{x}_{0}$ in the frame $\mathcal{E}$ is enough distant from material bodies, one may assume that $p_{1}\left(T_{0}, \mathbf{x}_{0}\right)=0$.

\section{Conclusion}

1) Differential identities show that, in standard electrodynamics, the number of the independent scalar PDE's is equal to the number of the unknown fields. This is true in the case that the 4-current $\mathbf{J}$ is assumed given. It is true also in the general case for which, instead, $\mathbf{J}$ belongs to the unknowns, i.e., when the motion of the charged continuum is not known a priori.

2) The same is true in the investigated theory of gravity ("SET"), if one sets the additivity assumption (1), i.e. $\boldsymbol{T}_{\text {inter }}=\mathbf{0}$ in Eq. (2). That assumption, however, leads to an untenable production or destruction of electric charge. Thus one must have in general $\boldsymbol{T}_{\text {inter }} \neq \mathbf{0}$, so the form of $\boldsymbol{T}_{\text {inter }}$ has to be precised, necessarily involving additional field(s). As a consequence, the former equations of electrodynamics of SET are not a closed system any more and must be supplemented by some new equation(s). 
3) In order for $\boldsymbol{T}_{\text {inter }}$ to be Lorentz-invariant in SR, it must involve just a scalar field $p$ and have the form (25). Thus, just one more equation is needed, and it can consistently be imposed to be the charge conservation, Eq. (26). The additivity (1) of the energy tensors is then recovered for a constant gravitational field, and hence in particular in the situation of SR.

4) In a weak and slowly varying gravitational field, and with a given EM field, the charge conservation (26) rewrites as an advection equation with given source for the scalar field $p$, Eq. (31). Hence $p$ may be calculated by integration along characteristics, Eq. (36). The corresponding interaction energy $T_{\text {inter }}^{00}$ could be counted as "dark matter", since: (i) it is necessarily present as soon as there is matter that emits an EM field; (ii) it is not especially localized inside that matter; (iii) it is gravitationally active in SET, as is any form of material energy.

Acknowledgement: I am grateful to Jerzy Kijowski for a discussion on the motion of the sources.

\section{References}

[1] Arminjon M., Scalar Theory of Gravity as a Pressure Force, Rev. Roum. Sci. Tech.- Méc. Appl. 42 (1997) 27-57.

[2] Arminjon M., On the Possibility of Matter Creation/Destruction in a Variable Gravitational Field, Anal. Univ. Bucureşti - Fizică 47 (1998) 3-21.

[3] Arminjon M., Accelerated Expansion as Predicted by an Ether Theory of Gravitation, Phys. Essays 14 (2001) 10-32.

[4] Arminjon M., Gravity as Archimedes' Thrust and a Bifurcation in that Theory, invited contribution to Franco Selleri's Festschrift (G. Tarozzi \& A. van der Merwe, eds.), Found. Phys. 34 (2004) 1703-1724.

[5] Arminjon M., Space Isotropy and Weak Equivalence Principle in a Scalar Theory of Gravity, Braz. J. Phys. 36 (2006) 177-189.

[6] Arminjon M., Summary of a Non-Uniqueness Problem of the Covariant Dirac Theory and of Two Solutions of It, In: Geometry, Integrability and Quantization XIV, I. Mladenov, A. Ludu and A. Yoshioka (Eds), Avangard Prima, Sofia 2013, pp 48-60. 
[7] Arminjon M., Continuum Dynamics and the Electromagnetic Field in the Scalar Ether Theory of Gravitation, Open Physics 14 (2016) 395-409.

[8] Arminjon M., Charge Conservation in a Gravitational Field in the Scalar Ether Theory, Open Physics 15 (2017) 877-890.

[9] Arminjon M., On the Equations of Electrodynamics in a Flat or Curved Spacetime and a Possible Interaction Energy, Open Physics 16 (2018) 488-498.

[10] Arminjon M., Lorentz-Invariant Second-Order Tensors and an Irreducible Set of Matrices, Submitted for publication, and Preprint HAL-01797592 (2018).

[11] Cattaneo C., General Relativity: Relative Standard Mass, Momentum, Energy and Gravitational Field in a General System of Reference, Nuovo Cim. 10 (1958) 318-337.

[12] Das A., The Special Theory of Relativity - a Mathematical Exposition, 2nd Printing, Springer-Verlag, Berlin - Heidelberg - New York 1996.

[13] Jiang B.N., Wu J., Povinelli L.A., The Origin of Spurious Solutions in Computational Electromagnetics, J. Comput. Phys. 125 (1996) 104-123.

[14] Landau L.D., Lifshitz E.M., The Classical Theory of Fields, 3rd English Edition, Pergamon, Oxford 1971.

[15] Liu C., Explanation on Overdetermination of Maxwell's Equations, Physics and Engineering (in Chinese) 27 (2017) 7-9, and Preprint arXiv:1002.0892v9 (2018).

[16] Misner C.W., Thorne K.S., Wheeler J.A., Gravitation, Freeman, San Francisco 1973.

[17] Møller C., The Theory of Relativity, Clarendon Press, Oxford 1952.

[18] Zhou X.L., On Independence, Completeness of Maxwell's Equations and Uniqueness Theorems in Electromagnetics, Prog. Electromagn. Res. 64 (2006) $117-134$. 